\title{
Intervenção fonoaudiológica e defficiência visual: percepções de profissionais de equipe interdisciplinar
}

\section{Hearing and speech patology intervention \& visual impairment: perceptions of professionals of staff cross-disciplinary}

Mayla M. B. Monteiro ${ }^{1}$, Rita C. I. Montilha ${ }^{2}$

\begin{abstract}
RESUMO
Objetivo: verificar conhecimento e opinião de profissionais da área da saúde e da educação acerca da intervenção fonoaudiológica com portadores de deficiência visual. Método: realizou-se estudo descritivo com profissionais da área da saúde e educação que atuam no Centro de Estudos e Pesquisa em Reabilitação "Prof. Dr. Gabriel O. S. Porto" (Cepre) na habilitação/reabilitação de portadores de deficiência visual. Utilizou-se um questionário auto-aplicável, desenvolvido após estudo exploratório e testeprévio. Resultados: a amostra foi composta por 21 profissionais da área da saúde e educação. A média de idade foi de 42.5 anos; $95,2 \%$ são do sexo feminino. Em relação às características profissionais as categorias de Serviço Social e Terapia Ocupacional predominaram com $33,3 \%$ cada, seguida de Psicologia $19,0 \%$. Os principais atendimentos realizados pela Fonoaudiologia conhecidos pelos profissionais que participaram do estudo, referem-se a aspectos de motricidade oral e alterações de fala e linguagem (38,0\% em ambos). Em relação à inserção da Fonoaudiologia no processo de estimulação e/ou reabilitação os profissionais apontaram como sendo o desenvolvimento global $(38,0 \%)$ o principal aspecto em que a Fonoaudiologia poderia contribuir. Conclusão: percebe-se que há conhecimento da atividade fonoaudiológica por parte dos profissionais que atuam na habilitação/reabilitação de portadores de deficiência visual, porém este ainda é restrito. Sugere-se o desenvolvimento de outros estudos sobre a intervenção da Fonoaudiologia na deficiência visual a fim de fornecer subsídios teóricos para sua atuação.
\end{abstract}

Palavras-chave: Reabilitação. Portadores de Deficiência Visual. Equipe Interdisciplinar de Saúde. Fonoaudiologia.

\section{Introdução}

A Fonoaudiologia é a ciência que estuda os distúrbios da comunicação humana, ou seja, as altera- ções de fala, linguagem, voz, audição, órgãos fonoarticulatórios e funções neurovegetativas (mastigação, sucção, deglutição e respiração). A atuação do profissional da área pode ser feita em escolas, hospitais,
1. Fonoaudióloga; Mestranda do Programa "Saúde, Interdisciplinaridade e Reabilitação" do Centro de Estudos e Pesquisa em Reabilitação "Prof. Dr. Gabriel O. S. Porto" - Faculdade de Ciências Médicas - UNICAMP

2. Docente do Curso de Fonoaudiologia - UNICAMP e do Centro de Estudos e Pesquisa em Reabilitação "Prof. Dr. Gabriel O. S. Porto" - Faculdade de Ciências Médicas - UNICAMP
CORRESPONDÊNCIA: Mayla M. B. Monteiro Rua Tessalia Vieira de Camargo, 126 13084-971 Campinas-SP email: mamyrina@ @cm.unicamp.br 
clínicas particulares, centros de saúde, indústrias, instituições, teatros, televisão, empresas de aparelhos

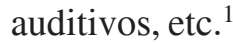

Esta ciência busca prevenir a ocorrência dos distúrbios mencionados anteriormente, diagnosticar e tratar de indivíduos portadores dos mesmos, minimizando as sequelas de tais distúrbios, promovendo a integração da forma mais eficiente possível à sociedade. $^{1}$

Na reabilitação de pessoas com deficiência visual, vários profissionais estão envolvidos. Com as descrições apresentadas acima, o fonoaudiólogo mostra que também poderá contribuir para este processo, visto que é um profissional que trabalha com diversas áreas, visando à integralidade das mesmas.

Adota-se como conceito de deficiência visual a presença de cegueira ou baixa visão. Segundo World Health Organization ${ }^{2}$ é considerado portador de cegueira o indivíduo com acuidade visual desde $3 / 60(0,05)$, no olho de melhor visão e melhor correção óptica possível, até a ausência de percepção de luz, ou correspondente perda de campo visual no olho de melhor visão com melhor correção possível. ${ }^{2}$ A baixa visão corresponde a um comprometimento de seu funcionamento visual mesmo após tratamento e/ou correção de erros refracionais comuns e apresenta uma acuidade visual inferior a 6/18 até a percepção de luz ou num campo visual inferior à 10 graus do seu ponto de fixação mas que utiliza ou é potencialmente capaz de utilizar a visão para o planejamento e execução de uma tarefa. ${ }^{2}$

O primeiro ano de vida é crítico para o desenvolvimento das funções visuais e a privação de estímulos visuais durante esse período pode provocar alterações anatômicas e funcionais irreversíveis. ${ }^{3}$

A visão desempenha papel fundamental nos primeiros anos de vida, pois é um estímulo motivador para a comunicação e a realização das ações. Considerando que, nesses anos o relacionamento com o mundo exterior é feito em grande parte por meio da visão, qualquer anormalidade nos olhos ou em sua função pode levar à dificuldades no aprendizado e no relacionamento social, além de uma diminuição no rendimento das atividades físicas e intelectuais ${ }^{3}$.

A ausência da visão limita a motivação espontânea para os deslocamentos gerando atrasos nas aquisições de habilidades manuais, posturais e de movimentos. Atrasos significativos decorrem da manipulação e da exploração precária de objetos, geradas pela dificuldade de integrar as informações captadas pelos outros sentidos. A limitação na experimentação ativa compromete a descoberta do mundo e, em consequência, a aquisição de conhecimentos. ${ }^{4}$

Crianças com baixa visão possuem necessidades especiais relacionadas ao uso de sua visão residual. Ela pode restringir, por exemplo, a experiência de vida da criança, a sua velocidade de trabalho, o desenvolvimento motor, a orientação e mobilidade e a capacidade de realizar atividades práticas. Pode também afetar sua educação assim como o desenvolvimento social e emocional. Existe um consenso de que muitas crianças têm condições de aprender e frequentar escolas comuns alternando a obtenção de informações tanto de forma visual como de forma tátil. Crianças com baixa visão não necessitam e não deveriam ser educadas como se fossem totalmente cegas. Portanto, é grande a necessidades de se incentivar o uso eficiente da baixa visão através de um programa de estimulação da visão residual. ${ }^{2}$

O som desempenha importante papel na estimulação e na relação das crianças cegas como o meio externo. O bebê aprende gradualmente a reconhecer os diferentes significados dos barulhos produzidos pela sua mãe e passa a reagir para estes sons com mudanças no comportamento e respostas vocais dele próprio. ${ }^{5}$

A audição constitui uma as principais áreas da Fonoaudiologia. Deste modo, o atendimento fonoaudiológico de pessoas com deficiência visual deve incluir estimulação e treino de sons, para fins de orientação, mobilidade e proteção.

Para a aquisição da linguagem, sabe-se o quão importante é a criança estar exposta a visão. A criança vidente observa as atividades que a mãe realiza e estabelece a relação entre os objetos. Frequentemente, essas ações são acompanhadas pela linguagem oral, gestual, expressões faciais, contato de olho, sorriso, entonação. ${ }^{4}$

Estudos realizados com mães de crianças cegas demonstram que essas mães, quando comparadas com mães de bebês videntes, têm uma tendência a falar menos com seus bebês. Este fato poderia explicar o comportamento passivo, geralmente observado em bebês cegos e o aparecimento tardio de vocalizações para o início dos contatos. É por meio da linguagem que, muitas vezes, o cego aprende o mundo. ${ }^{6}$

A Fonoaudiologia compreende que as ações que para os videntes são de aprendizado "natural", como levar um talher à boca, manter o posicionamento da cabeça direcionado a quem dirige a fala, interagir com 
uma pessoa por meio de gestos, dar ênfase ao conteúdo do contexto de fala, manter um diálogo com pessoas desconhecidas; para as pessoas com deficiência visual é um aprendizado não muito simples. ${ }^{7}$

Visando uma melhora nas condições de hábitos orais e de comunicação, a Fonoaudiologia pode e deve ser inserida dentro de um processo de habilitação/reabilitação de portadores de deficiência visual.

Os profissionais que atuam na reabilitação e educação de indivíduos deficientes visuais necessitam deter conhecimentos sobre as limitações destes, bem como sobre o sistema de ensino e reabilitação vigentes. ${ }^{8}$

Reconhece-se no mundo todo, a necessidade de identificar indivíduos com afecções oculares. O dimensionamento de aspectos dessa problemática, por meio da investigação científica, provê base concreta para o planejamento de ações curativas e preventivas relacionadas à saúde ocular. ${ }^{9} \mathrm{O}$ processo de reabilitação visa auxiliar portadores de deficiência visual em atividades rotineiras, nas quais agora encontra dificuldade em realizar. Este processo deve favorecer a expressão de sentimentos, a espontaneidade, o conhecimento das potencialidades e limitações e o desenvolvimento e aprimoramento de habilidades. As atividades desenvolvidas podem ser expressivas, pedagógicas, lúdicas, de automanutenção e profissionalizantes. ${ }^{9}$

Sabe-se que hoje em dia, a proposta reabilitacional visa à integração de diferentes áreas na intervenção terapêutica, saindo de uma visão fragmentária para uma concepção unitária do ser humano.

A interdisciplinaridade representa o grau mais avançado de relação entre disciplinas, considerandose o critério de real entrosamento entre elas. Nesse caso, seriam estabelecidas relações menos verticais entre diferentes disciplinas, que passariam, também, a compartilhar uma mesma plataforma de trabalho, operando sob conceitos em comum e esforçando-se para decodificar o seu jargão para os novos colegas, ${ }^{10}$ que no caso deste estudo compreende o processo de reabilitação.

O melhor uso da baixa visão envolve a adaptação de instrumentos de apoio tais como recursos especiais (ópticos ou não ópticos). A maioria dos recursos ópticos é composta por lentes que aumentam a imagem (lupa, telelupa, óculos comum). Já os não ópticos são recursos simples que tem a finalidade de aumentar a resolução visual, como canetas hidrográficas de ponta grossa, iluminação variável, material com impressão ampliada e microcomputadores. Além disso, profissionais foram e estão sendo treinados para ensinarem deficientes visuais a utilizarem esses recursos durante a reabilitação. ${ }^{10}$

A sociedade arca com prejuízos em decorrência da falta de atenção com a saúde visual, representados pela diminuição de produtividade, da sua força de trabalho e o elevado custo de ações de reabilitação. Acrescem-se a isto, consequências psicológicas, sociais e econômicas para o deficiente visual devido às restrições ocupacionais, diminuição da renda, perda de "status", de auto-estima, de auto-confiança. Desse modo, a qualidade de vida é afetada, como tem sido observado especialmente em países em desenvolvimento. ${ }^{11}$

Este estudo teve por objetivo verificar conhecimento e opinião de profissionais da área da saúde e da educação acerca da intervenção fonoaudiológica de portadores de deficiência visual.

\section{Materials e Métodos}

A amostra deste estudo foi constituída por profissionais da área da saúde e educação (assistentes sociais, terapeutas ocupacionais, fisioterapeutas, fonoaudiólogos, psicólogos, pedagogos) que atuam no Centro de Estudos e Pesquisa em Reabilitação "Prof. Dr. Gabriel O. S. Porto" (Cepre) na habilitação/reabilitação de portadores de deficiência visual e que assinaram o Termo de Consentimento Livre Esclarecido. Foram excluídos da pesquisa profissionais que não atuavam no Centro de Estudos e Pesquisa em Reabilitação "Prof. Dr. Gabriel O. S. Porto" (Cepre) e/ou na habilitação/reabilitação de pessoas com deficiência visual, além dos profissionais que participaram do estudo exploratório e do teste prévio.

As variáveis deste estudo referiram-se à profissionais da área da saúde e educação que atuassem na habilitação/reabilitação de portadores de deficiência visual. Optou-se por agrupá-las desta forma:

Características pessoais e profissionais [Sexo, Idade, Ocupação, Categoria Profissional, tempo de atuação na habilitação/reabilitação de portadores de deficiência visual, tipo de Instituição em que atua (Organizações Não Governamentais, Instituição de Ensino Superior, Instituição Governamental), faixa etária com a qual atua, vínculo com a instituição (profissional, estagiário, aprimorando, docente), atuação ou não com equipe interdisciplinar].

Aspectos referentes à percepção de profissionais acerca da intervenção fonoaudiológica na 
habilitação/reabilitação de pessoas com deficiência visual (Conhecimento acerca da intervenção fonoaudiológica na habilitação/reabilitação de pessoas com deficiência visual, opinião acerca da intervenção fonoaudiológica na habilitação/reabilitação de pessoas com deficiência visual).

Foi realizado estudo exploratório para a elaboração de instrumento de medida. Primeiramente foram realizadas entrevistas abertas, em que foram abordados temas sobre o conhecimento e opinião dos profissionais acerca da intervenção fonoaudiológica na habilitação/reabilitação de portadores de deficiência visual. As entrevistas foram realizadas no Cepre devido a viabilidade de acesso da amostra.

Com finalidade de aperfeiçoar a forma do instrumento, foi realizado o pré-teste que intrega-se gradualmente às etapas do estudo exploratório. O estudo exploratório constitui prova necessária para assegurar confiança e validade dos dados a serem obtidos na pesquisa. ${ }^{13}$

Neste estudo, o pré-teste foi realizado aplicando-se o questionário estruturado a profissionais que atuam na habilitação/reabilitação de portadores de deficiência visual do Cepre. O pré-teste, também foi realizado no Cepre devido a viabilidade de acesso à amostra.

O questionário foi desenvolvido baseado nos objetivos e nas variáveis apresentadas no projeto, que foram apresentadas anteriormente.

A coleta de dados foi realizada por meio de questionário auto-aplicável com profissionais que aceitaram participar da pesquisa. As entrevistas foram realizadas individualmente, respeitando-se o local de atuação do profissional no serviço.

Antes de iniciar a aplicação do questionário com cada profissional foram explicados os objetivos da pesquisa, assim como foram assegurados o anonimato e a confidencialidade das informações, por meio de assinatura de um termo de consentimento livre e esclarecido.

As variáveis foram categorizadas a fim de suprir os objetivos da pesquisa. Para a apresentação dos resultados, foram apresentadas tabelas de distribuição de frequência, os dados foram agrupados em classes de modo a fornecer valores absolutos e percentagem, não tendo sido utilizados outros testes estatísticos.

O projeto foi aprovado pelo Comitê de Ética em Pesquisa da Faculdade de Ciências Médicas da Unicamp, CAAE 0209.0.146.000-06.

\section{Resultados}

A amostra deste estudo foi composta por 21 profissionais da área da saúde e educação.

A distribuição por sexo apresentou-se consideravelmente maior no sexo feminino. Em relação às características profissionais a categoria de terapeuta ocupacional foi a que mais apareceu $(33,3 \%)$, seguida de assistente social 19\% (Tabela 1).

A maioria da amostra respondeu que o tipo de vínculo que possui com a instituição é o de docente $(57,1 \%)$ (Tabela 1$)$.

Os profissionais da amostra relataram que atuam com pessoas com deficiência visual entre 6 meses e 10 anos.

\section{Tabela 1}

Características profissionais

\begin{tabular}{lrr}
\hline & & $\mathrm{N}=21$ \\
\hline Características Profissionais & $f$ & $\%$ \\
\hline Categoria Profissional & & \\
$\quad$ Terapia Ocupacional & 7 & 33,3 \\
$\quad$ Serviço Social & 4 & 19,0 \\
Pedagogia & 3 & 14,2 \\
Psicologia & 3 & 14,2 \\
Pedagogia Especializada em DV & 2 & 9,5 \\
Fisioterapia & 1 & 4,8 \\
Fonoaudiologia & 1 & 4,8 \\
TOTAL & 21 & 100
\end{tabular}

Tipo de vínculo com a Instituição

$\begin{array}{lrr}\text { Docente } & 12 & 57,1 \\ \text { Aprimorando } & 4 & 19,0 \\ \text { Estagiário } & 4 & 19,0 \\ \text { Profissional } & 1 & 4,8 \\ \text { TOTAL } & 21 & 100\end{array}$

Em relação à existência de atendimento fonoaudiológico na equipe de atuação, a maioria referiu que há fonoaudiólogos compondo a equipe interdisciplinar na habilitação/reabilitação de portadores de deficiência visual. Em relação ao tipo de atendimento desenvolvido pelo fonoaudiólogo, $72,7 \%$ dos profissionais referiam que o fonoaudiólogo realiza avaliação e atendimentos regulares (Tabela 2). 


\section{Tabela 2}

Existência e tipo de trabalho fonoaudiológico na equipe de atuação

\begin{tabular}{lrr}
\hline & & $\mathrm{N}=21$ \\
\hline Existência & $f$ & $\%$ \\
\hline Sim & 12 & 57,1 \\
Não & 9 & 42,8 \\
TOTAL & 21 & 100,0 \\
& & $\mathrm{~N}=12$ \\
\hline Tipo & $f$ & $\%$ \\
\hline Avaliação e atendimento & & \\
fonoaudiológico & 8 & 66,6 \\
Estimulação & 2 & 16,7 \\
Teste da orelhinha & 2 & 16,7 \\
TOTAL & 12 & 100,0 \\
\hline
\end{tabular}

As principais atividades realizadas pela fonoaudiologia conhecidas por estes profissionais que responderam ao questionário referiram-se a aspectos de motricidade oral e alterações de fala e linguagem (38\% em ambos) (Tabela 3).

Os profissionais responderam como sendo a fala e linguagem $(52,3 \%)$ seguidos de motricidade oral $(33,3 \%)$, os aspectos que conheciam, referentes a atua-

\section{Tabela 3}

Conhecimento do funcionamento do trabalho realizado pela Fonoaudiologia durante a estimulação e/ou reabilitação

\begin{tabular}{lrr}
\hline & & $\mathrm{N}=21$ \\
\hline Conhecimento* & $f$ & $\%$ \\
\hline Aspectos de motricidade oral & 8 & 38,0 \\
Alterações no processo de aquisição de & & \\
linguagem e fala & 8 & 38,0 \\
Avaliação e Intervenção fonoaudiológica & 4 & 19,0 \\
Orientações à família & 4 & 19,0 \\
Diagnóstico de perda auditiva & 4 & 19,0 \\
Expressões faciais & 2 & 9,6 \\
Não conhece & 5 & 23,8 \\
TOTAL & 35 & 100,0 \\
\hline
\end{tabular}

*Respostas múltiplas ção da Fonoaudiologia, que poderiam auxiliar portadores de deficiência visual. Destaca-se também a proporção de profissionais que relataram não conhecer os aspectos em que a Fonoaudiologia poderia auxiliar estas pessoas $(33,3 \%)$

Em relação à percepção de melhoras ao final da estimulação e/ou reabilitação, os profissionais apontaram uma melhora global no processo $(28,5 \%)$, seguido do desenvolvimento linguístico $(19,0 \%)$. Destaca-se também a proporção de profissionais que relataram não saber se ocorreram melhoras ou não no aspecto fonoaudiológico ao final da estimulação $(38,0 \%)$ (Tabela 4).

\section{Tabela 4}

Percepções de melhoras ao final da estimulação e/ou reabilitação no aspecto fonoaudiológico

\begin{tabular}{lrr}
\hline & & $\mathrm{N}=21$ \\
\hline Percepções* & $f$ & $\%$ \\
\hline Melhora global & 6 & 28,5 \\
Desenvolvimento lingüístico & 4 & 19,0 \\
Alterações de motricidade oral & 3 & 14,2 \\
Relação com a família & 2 & 9,5 \\
Problemas auditivos & 1 & 4,8 \\
Expressão facial & 1 & 4,8 \\
Não sabe & 8 & 38,0 \\
TOTAL & 25 & 100,0 \\
\hline
\end{tabular}

*Respostas múltiplas

A maioria opinou como sendo muito importante $(47,6 \%)$ a intervenção fonoaudiológica a portadores de deficiência visual (Tabela 5).

\section{Tabela 5}

Opinião acerca da importância da intervenção fonoaudiológica com portadores de deficiência visual

\begin{tabular}{lrr}
\hline & & $\mathrm{N}=21$ \\
\hline Opinião & $f$ & $\%$ \\
\hline Muito importante & 10 & 47,6 \\
Importante & 7 & 33,4 \\
Nada importante & - & - \\
Não sabe/ Não conhece & 4 & 19,0 \\
TOTAL & 21 & 100,0 \\
\hline
\end{tabular}


Em relação à inserção da Fonoaudiologia no processo de estimulação e/ou reabilitação os profissionais destacaram como sendo o desenvolvimento global (38\%) o principal aspecto em que a Fonoaudiologia poderia auxiliar.

Em relação à Fonoaudiologia como sendo um auxiliar no aprimoramento/desenvolvimento de outros aspectos do processo de estimulação e/ou reabilitação, os profissionais opinaram como a melhora na leitura e escrita o principal auxílio (33,3\%), seguido da melhora da comunicação $(23,8 \%)$ (Tabela 6$)$.

\section{Tabela 6}

Opinião acerca de como a Fonoaudiologia pode auxiliar no aprimoramento/desenvolvimento de outros aspectos do processo de estimulação e/ou reabilitação

\begin{tabular}{lrr}
\hline & & $\mathrm{N}=21$ \\
\hline Opinião* & $f$ & $\%$ \\
\hline Melhora da leitura e da escrita & 5 & 23,3 \\
Melhora da comunicação & 4 & 19,0 \\
Melhora da fala & 3 & 14,2 \\
Orientação à família & 2 & 9,5 \\
Postura & 2 & 9,5 \\
Estimulação do resíduo visual & 2 & 9,5 \\
Atividade lúdica & 2 & 9,5 \\
Qualidade de vida & 2 & 9,5 \\
Inserção social & 1 & 4,8 \\
Estimulação & 1 & 4,8 \\
Percepção auditiva & 1 & 4,8 \\
Melhora da auto-estima & 1 & 4,8 \\
Expressões faciais & 3 & 14,2 \\
Não sabe & 36 & 100,0 \\
TOTAL & & \\
\hline
\end{tabular}

* Respostas Múltiplas

\section{Discussão}

A investigação foi realizada por meio de "survey". O "survey" constitui modalidade de pesquisa frequentemente empregada no setor da saúde. Trata-se de um tipo de investigação científica em que se estuda a realidade de forma como se apresenta, sem o propósito de estabelecer relação causal entre as variáveis $^{13}$.

A maior parte dos sujeitos que respondeu ao questionário é formada por profissionais de diferentes categorias da área da saúde. Percebe-se então que a interdisciplinaridade está cada vez mais presente dentro das Instituições. A interdisciplinaridade assume grande importância na medida em que identifica e nomeia uma mediação possível entre saberes e competências e garante a convivência criativa com as diferenças. Além da função de mediador, o conceito de interdisciplinaridade vem apontar a insuficiência dos diversos campos disciplinares, abrindo caminhos e legitimando o tráfego de sujeitos concretos e de conceitos e métodos entre as diferentes áreas do conhecimento $^{10}$.

Quando questionados sobre sua ocupação, a maioria dos profissionais respondeu como sendo docentes $(61,9 \%)$. Isto se deve ao fato de que o Cepre está inserido em uma Instituição de Ensino Superior ; e a maioria de seus profissionais serem docentes de curso de graduação e especialização, além de atuarem diretamente na assistência de pessoas com deficiência visual.

Neste trabalho encontramos um predomínio de profissionais que atuam com deficiência visual há uma década $(57,9 \%)$. No Brasil, a reabilitação visual de pessoas com baixa visão surgiu em 1974, com a criação das primeiras clínicas de treinamento de visão subnormal ${ }^{15}$. Mostra-se assim que os profissionais que atuam na habilitação/reabilitação da deficiência visual estão atuando há um período relativamente próximo ao início da reabilitação de deficientes visuais no Brasil.

Quando questionados sobre a existência de atividades fonoaudiológicas na sua equipe de atuação, a maioria dos profissionais respondeu positivamente $(57,1 \%)$. E quando questionados quanto ao tipo de intervenção, a resposta mais frequente foi a de que havia avaliação e atendimento fonoaudiológico $(72,7 \%)$. Este dado aponta que em certos lugares a Fonoaudiologia está sendo inserida no processo de habilitação e/ ou reabilitação de pessoas com deficiência visual. Porém esta inserção ainda é pequena, visto que mesmo dentro do Cepre, onde há uma equipe interdisciplinar atuando na reabilitação de pessoas com deficiência visual, apenas um fonoaudiólogo atua diretamente e, alguns outros profissionais, inseridos no serviço, referiram desconhecer a atuação da Fonoaudiologia na 
deficiência visual. Pode-se associar este fato à escassez de literatura disponível em relação essa temática, como já foi explicitado anteriormente.

Observam-se que proporção acentuada dos entrevistados $(66,6 \%)$ revelaram conhecimento sobre intervenção fonoaudiológica junto aos deficientes visuais. No entanto, as autoras tiveram dificuldade em categorizar as respostas, por não haver domínio da nomenclatura apropriada. Como exemplo, alguns profissionais citaram como posicionamento de língua e alimentação, alguns dos aspectos trabalhados pela Fonoaudiologia. Estes dois aspectos se enquadram na área de motricidade oral, mas não foram assim apontados por parte dos profissionais que responderam ao questionário. Em relação à alimentação, o profissional que atua junto à pessoa com deficiência visual, deve verificar a iluminação do ambiente, a possibilidade de os utensílios oferecerem contrastes entre si, os copos e xícaras em relação aos líquidos e o alimento em relação ao prato. A Fonoaudiologia junto com a terapia ocupacional pode auxiliar o paciente na melhor forma para ele se alimentar. A Fonoaudiologia indicaria os melhores alimentos, melhor posição para a realização da refeição, e a melhor utilização dos músculos da mastigação e deglutição com o intuito de coordenar a dinâmica da alimentação.

Os principais conhecimentos relacionados as atividades realizadas pela Fonoaudiologia durante a estimulação e/ou reabilitação foram referentes às alterações de motricidade oral (38\%) e no processo de aquisição de linguagem e fala (38\%). O diagnóstico tardio da deficiência visual priva a criança de receber a estimulação necessária ao seu desenvolvimento. Por outro lado, as crianças com baixa visão que recebem intervenção precoce têm maiores possibilidades de desenvolver seu resíduo visual. A intervenção precoce melhora a qualidade de vida da criança com baixa visão. Deste modo, tão logo seja feito diagnóstico, os pais devem ser orientados sobre a importância da intervenção precoce para o desenvolvimento da criança. $^{4}$

Sabe-se da importância de projetos de triagem visual em crianças, sejam elas normais ou com múltiplas deficiências, uma vez que seu tratamento e acompanhamento deve ser o mais precoce possível, dentro do período da plasticidade visual, favorecendo a reabilitação visual, o que estimula o desenvolvimento global da criança ${ }^{16}$.

Com relação ao conhecimento de quais aspectos em que a Fonoaudiologia poderia auxiliar pessoas com deficiência visual, a maioria dos profissionais apontou a fala e a linguagem $(52,3 \%)$, seguida pela motricidade oral $(33,0 \%)$. A linguagem e a fala atuam como controladoras do ego sobre os afetos e impulsos, aumentando a possibilidade de distinção entre desejos e fantasias de um lado e a realidade do outro. No cego, além de desempenharem estas funções, a linguagem e a fala são usadas também como substitutos de "coisas" que não vê: descobre usos da fala para se orientar, para catalogar características que diferenciam as pessoas, para descobrir alguma marca pela qual um objeto possa ser reconhecido ${ }^{14}$.

O dado citado acima aponta a Fonoaudiologia como ciência auxiliadora de portadores de deficiência visual na melhora de seu desempenho de fala e linguagem visando uma comunicação mais efetiva.

Apesar de um número diversificado de exemplos apontados pelos profissionais, alguns ainda não conhecem os aspectos em que a Fonoaudiologia poderia auxiliar as pessoas com deficiência visual, mesmo estando inseridos em Centro onde há um curso de Graduação em Fonoaudiologia. Este dado poderia sugerir que o profissional não procura por este conhecimento ou não sente necessidade do mesmo durante o processo de reabilitação no qual atua. Sem base teórica, o profissional não consegue perceber em que a Fonoaudiologia poderia levar seus conhecimentos e aprimorar a estimulação/reabilitação de pessoas com deficiência visual.

A melhora global dos pacientes $(28,5 \%)$ ressalta-se na questão sobre as percepções de melhoras ao final da estimulação e/ou reabilitação no aspecto fonoaudiológico. A Fonoaudiologia, como uma ciência recente, carece de estudos que possam produzir conhecimentos técnicos e científicos capazes de expandir seu domínio de atuação em saúde pública ${ }^{17}$.

Faz-se relevante que a Fonoaudiologia acompanhe as mudanças teórico-metodológicas do campo da Saúde Pública/Coletiva e, pautando-se por elas, participe do processo de implantação de uma política de saúde nacional, definindo seu papel e lugar junto à Promoção da Saúde da população de maneira reflexiva, consciente, responsável e atuante ${ }^{18}$. Dentre elas, pode-se citar a atuação Fonoaudiológica na deficiência visual.

Por não conhecerem totalmente as áreas de atuação da Fonoaudiologia e nem todos os seus aspectos, $38,0 \%$ dos profissionais não sabiam responder a esta questão, e relataram como sendo uma melhora global sua percepção em relação ao aspecto fonoau- 
diológico. Isto sugere que os outros profissionais que atuam em reabilitação de pessoas com deficiência visual não possuem conhecimento a respeito da Fonoaudiologia. Observa-se que são raros os trabalhos na literatura que abordem essa temática. Sugere-se o desenvolvimento de outros estudos sobre a intervenção da Fonoaudiologia na deficiência visual a fim de fornecer subsídios teóricos para sua atuação, além da sua inserção nos processos de habilitação/reabilitação em deficiência visual.

Este dado sugere que esta pode ser uma nova área em que o fonoaudiólogo poderá se inserir, trazendo seus conceitos para auxiliar no aprimoramento do processo de reabilitação de portadores deficiência visual.

A respeito da opinião da importância da intervenção fonoaudiológica em portadores de deficiência visual, a maioria dos profissionais consideram a Fonoaudiologia muito importante na habilitação/reabilitação de pessoas com deficiência visual $(47,6 \%)$. Mesmo sabendo que o trabalho integrado da Fonoaudiologia na estimulação/reabilitação de pessoas com deficiência visual é importante para uma visão integral do paciente, os profissionais que atuam em equipe de reabilitação geralmente só solicitam intervenção fonoaudiológica quando há queixa por parte do paciente ou da família ou se na opinião deles o aspecto no qual os pacientes apresentam alterações diz respeito à Fonoaudiologia.
A melhora da leitura e da escrita $(33,0 \%)$, seguida da melhora da comunicação $(23,8 \%)$ foram apontados como os principais aspectos de como a Fonoaudiologia pode auxiliar no aprimoramento/desenvolvimento de outros aspectos do processo de estimulação e/ou reabilitação. A comunicação escrita através da leitura e da escrita propriamente dita, reflete $56,0 \%$ e $52,0 \%$, respectivamente, das limitações segundo a opinião de portadores de deficiência visual do estudo. Dado que ressalva a importância de atuação fonoaudiológica para a melhora da comunicação e independência de pessoas com deficiência visual. ${ }^{17}$

\section{Conclusão}

Percebe-se que há conhecimento do trabalho fonoaudiológico por parte de profissionais de área da saúde e educação que atuam na habilitação/reabilitação de pessoas com deficiência visual, porém este ainda é restrito.

A maioria dos profissionais refere que são nos aspectos de motricidade oral e alterações de fala e linguagem os trabalhos da Fonoaudiologia conhecidos junto a deficientes visuais.

Opinaram como sendo a melhora na leitura e escrita e a melhora da comunicação as principais contribuições da Fonoaudiologia no aprimoramento/desenvolvimento de aspectos do processo de habilitação e/ou reabilitação de deficientes visuais.

\begin{abstract}
Purpose: To verify health and education's professional knowledge and opinion concerning Hearing and Speech Pathology intervention with visual impairment's person. Methods: A descriptive survey on health and education's professional that work at the Center of Studies an Researches in Rehabilitation "Prof. Dr. Gabriel O. S. Porto" (Cepre) at the habilitation/rehabilitation process of visual impairment's person. An self-applied questionnaire was used, desenveloped after a exploratory study and previous-test. Results: 21 health and education's professionals answered the questionnaire. The average age was 42.5 years; $92,5 \%$ are female. Related the professional's characteristics, the social service and occupation therapy category's appeared the most (33,3\% each), followed by psychology (19,0\%). The main works realized by the Hearing and Speech Pathology known by the professionals who answered the questionnaire were oroface-motricity's aspects and speech and language alteration's (38,0\% in both). Related to the Hearing and Speech Pathology's insertion in the stimulation and/or rehabilitation process, the professionals had pointed the global development $(38,0)$ the main aspect where the Hearing and Speech Pathology could contribute. Conclusion: There is knowledge about Hearing and Speech Pathology's work by the health and education's professionals that attended visual impairment's person, however it's still restricted. Others studies' development is suggested about the Hearing and Speech Pathology's intervention with the visual impairment in order to supply theoretical subsidies for its performance.
\end{abstract}

Keywords: Rehabilitation. Visually Impaired Persons. Patient Care Team. Speech, Language and Hearing Sciences. 


\section{Referências Bibliográficas}

1. Sacaloski M, Alavarsi E, Guerra GR. Fonoaudiologia na escola. São Paulo: Lovise; 2000. p. 53-5.

2. World Health Organization. Global date on blindness. Bulletin of the World Health Organization, 73.Genivè, WHO. 1995; 73(1):115-20.

3. Montilha RCI, Nobre MIRS, Gagliardo HGR. Atuação terapêutico-ocupacional junto a pacientes com transtornos da visão. In: Carlo MMRP, Luzo MCM, organizador. Terapia ocupacional: reabilitação física e contextos hospitalares. São Paulo: Roca; 2004. p. 276-91.

4. Botega MBS, Gagliardo HGRG. Intervenção precoce na deficiência visual: o que fazemos? Rev Soc Bras Fonoaudiol. 1998; 2(1):46-50.

5. Limongi SCO. Fonoaudiologia e pesquisa. São Paulo: Lovise; 1998. p.233-52.

6. Ochaita E, Rosa A. Percepção, ação e conhecimento nas crianças cegas. In: Coel C, Palácios G, Marchesi A. Desenvolvimento psicológico e educação. Porto Alegre: Artes Médicas; 1995. p.83-197.

7. Conselho Regional de Fonoaudiologia. Rev Fonoaudiologia. 2005; (62).

8. Montilha $\mathrm{RCl}$. O atendimento de terapia ocupacional com o adulto portador de cegueira adquirida. Sinopse de Oftalmologia. 2000; 2(1):24-5.

9. Montilha RCI, Temporini ER, Nobre MIRS, Gasparetto MERF, Kara-Jose N. Utilização de recursos ópticos e equipamentos por escolares com deficiência visual. Arq Bras Oftalmol. 2006 69(2):207-11
10. Furtado JP. Equipes de referência: arranjo institucional para potencializar a colaboração entre disciplinas e profissões. Interface: Comunicação, Saúde e Educação. 2007; 11 (22): 239-55.

11. Kara-Jose N, Temporini, ER. Cirurgia de catarata: o porquê dos excluídos. Rev Panamericana Salud Publica. 1999; 6(4):242-8.

12. Temporini IER, Kara-José N. Níveis de prevenção de problemas oftalmológicos: Propostas de intervenção. Arq Bras Oftalmol. 1995; 58:189-94.

13. Temporini ER. Saúde do escolar: conduta e opinião de professores do sistema de ensino do Estado de São Paulo. [tese]. São Paulo (SP): Universidade Estadual de São Paulo; 1986.

14. Amiralian MLTM. Psicodiagnóstio do cego congênito: aspectos cognitivos. [dissertação]. São Paulo (SP): Universidade de São Paulo; 1986

15. Montilha RCl. Reabilitação de portadores de deficiência visual: características, conhecimentos e opiniões de clientela atendida em um centro universitário. [dissertação]. Campinas (SP): Universidade Estadual de Campinas; 1997.

16. Ventura R, Ventura L, Carlos Brandt C, Ferraz D, Ventura B. Experiência em projeto: "Enxergando através das mãos". Arq Bras Oftalmol. 2007; 70(5): 823-6.

17. César AM, Maksud SS. Caracterização da demanda de fonoaudiologia no serviço público municipal de Ribeirão da Neves - MG. Rev CEFAC. 2007; 9(1):133-8.

18. Penteado RZ, Servilha EAM. Fonoaudiologia em saúde pública/coletiva: compreendendo a prevenção e o paradigma da promoção da saúde. Rev. Distúrbios da Comunicação, São Paulo, 16(1): 107-16, abril, 2004. 